\title{
Renal and Bleeding Complications in Critically Ill Covid-19 Patient-A Case Report
}

\author{
MI PIA ${ }^{\mathrm{a}}, \mathrm{T}$ ISLAM $^{\mathrm{b}}$
}

\begin{abstract}
Summary:
The ongoing outbreak of Covid-19 presented with a wide variety of clinical manifestations. Apart from the common respiratory complications, acute renal impairment and bleeding complications on full anticoagulation has been also observed in some patients. Here we report a 67 year old male with COPD and CKD presented with symptoms of covid-19 and found ground glass opacity on CT scan and bibasilar opacity on chest $X$-ray, admitted to the hospital and he was initially stable after supportive management, discharged home on antibiotics but readmitted after 4 days with worsening shortness of breath, hypoxia, tachycardia (A-fib with Rapid Ventricular Response) and high ESR. He was started on High flow nasal cannula (HFNC), diltiazem, adenosine and antibiotics ultimately needed intubation. While he was on antibiotics, hydroxychloroquine, DVT prophylaxis and statin he developed septic shock two days after intubation. Next day he had to receive Continuous Renal Replacement Therapy (CRRT).
\end{abstract}

He was placed on heparin infusion. With clinical improvement the patient was extubated to HFNC, but after

Case:

The patient is a 67 year old male with history of COPD (heavy smoker) and CKD who presented to the ED on $3 / 24 / 20$ for evaluation of fever and SOB (shortness of breath). Pt was found to have transient hypoxia ( $\mathrm{SpO} 2$ 80s on RA) and his CXR showed bibasilar opacities. CT imaging showed GGO (ground-glass opacity). Initial WBC 3.8, PCT 0.80. An RVP (Respiratory Viral panel) was negative. He was tested for COVID-19 earlier as an outpatient on $03 / 24 / 20$. He was given a dose of ceftriaxone and azithromycin, and subsequently admitted. His hospital stay was short and uneventfulhe was discharged home on 3/25/20 and prescribed

a. Masuma Islam Pia, Clinical Observer, Yale New Haven Hospital Connecticut, USA

b. Tasbirul Islam, Clinical Associate Professor, Indiana University of School of Medicine, Medical Director, Division of Pulmonary and Critical Care Medicine IU Arnett hospital, Lafayette, IN USA

Address of Correspondence: Tasbirul Islam, Clinical Associate Professor, Indiana University of School of Medicine, Medical Director, Division of Pulmonary and Critical Care Medicine, IU Arnett Hospital, Lafayette, IN USA, E-mail: Tislam@iuhealth.org; tasbirul@msn.com one day of extubation he developed bradycardia, hypotension and gradually became unresponsive. He was given vasopressors and intubated again. CT scan showed retroperitoneal hematoma $10 \times 7 \times 12 \mathrm{~cm}$. His heparin was discontinued and was managed conservatively. With supportive treatment his clinical condition improved gradually and was extubated again. CRRT was switched from CVVH (Continuous Veno-Venous Hemofiltration) to HD and eventually he was discharged home.

Clinicians should remain watchful at all stages of critical care management of COVID 19 because timely intervention and drug adjustment is lifesaving.

Keywords: COVID-19, Acute kidney injury (AKI), Retroperitoneal bleeding, Continuous renal replacement therapy (RRT).

(J Bangladesh Coll Phys Surg 2020; 38: 136-140) DOI: https://doi.org/10.3329/jbcps.v38i0.47341

Amoxicillin/clavulanate potassium for 5 days. He presented to the ED again on 3/29/20 for worsening SOB since discharge. Upon initial presentation he was tachycardic and hypoxic at $89 \%$, he was commenced on HFNC (High-flow nasal cannula oxygen). The monitor showed apparent a-fib w/ RVR(Rapid ventricular response) he did not respond to a $20 \mathrm{mg}$ diltiazem bolus. He was subsequently given adenosine, a second diltiazem bolus, started on a diltiazem drip, and readmitted to the PCU. ESR was initially 92. Upon admission, he was started on both azithromycin and hydroxychloroquine given his worsening COVID pneumonia. Piperacillin/tazobactam was also initiated in case of a secondary bacterial infection. Shortly after admission on $3 / 30 / 20$, he was showing increased respiratory distress requiring $\mathrm{HFNC}$ at $50 \mathrm{~L}$ w/ $\mathrm{FiO} 250 \%$ and ultimately ICU admission and intubation. On 3/30/ 20 shortly after intubation he was heavily sedated, proned, and paralyzed in accordance with ARDS protocol. He was started on DVT prophylaxis and a statin on 4/30/20 as well. On 4/2/20 he experienced septic shock requiring norepinephrine and vasopressin. Pt's renal function continued to decline requiring CCRT 
(Continuous renal replacement therapy) initiation on $4 /$ $4 / 20$ with $\mathrm{Cr} 4.85$. On $4 / 5 / 20$, the patient was switched to a heparin drip and on $4 / 6 / 20$ the statin was discontinued because of abnormal LFTs. On 4/8/20, he was given an extra $100 \mathrm{mg}$ of furosemide to facilitate SBT. He was subsequently extubated to HFNC. On 04/10/20 he experienced an episode of bradycardia, hypotension, and unresponsiveness lasting approximately 10-15 minutes. Given his decompensation, shock on vasopressors, and current condition he was re intubated. CT scan showed spontaneous retroperitoneal hematoma $10 \times 7 \times 12 \mathrm{~cm}$ requiring $3 \mathrm{PRBC}$ given his $\mathrm{Hb}$ was under 7 . CT imaging of the chest was remarkable for ground glass opacities throughout and a right pneumothorax for which chest tube was placed. His heparin drip was also discontinued on 4/10 given his spontaneous RP bleed. Echocardiography throughout his ICU stay remained consistent with preserved LV function. On 4/10 blood cultures were obtained and he was started empirically on vancomycin and cefepime for shock and leukocytosis with WBC 33. A repeat COVID swab on 4/11/20 was positive and prednisone was switched to Dexamethasone. He was extubated on 4/14 and switched from CVVH (Continuous Veno-Venous hemofiltration) to HD. He was eventually weaned to $2 \mathrm{~L} / \mathrm{min} \mathrm{NC}$. On $4 /$ $16 / 20$ hemodialysis was discontinued, and discharged home on 4/26/20. Medications administered during his stay include prednisone, azithromycin, hydroxychloroquine, vancomycin, cefepime, enoxaparin, heparin and Dexamethasone. Of note, the patient was not treated with Tocilizumab.

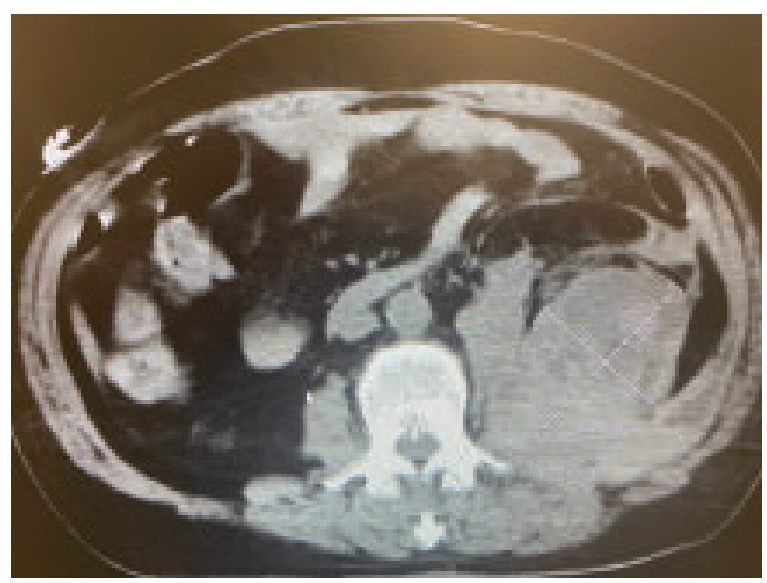

Retroperitoneal bleed.

\section{Discussion:}

Coronaviruses are a large family of viruses that can infect both humans and animals. The first coronavirus was found in chickens in the 1930s. Human coronaviruses were first identified in the mid 60's. To date, seven coronaviruses can cause disease in humans. SARS-CoV-2 is one of seven types of coronavirus, including the ones that cause severe diseases like Middle East respiratory syndrome (MERS) and sudden acute respiratory syndrome (SARS). COVID-19 has been declared a public health emergency on January $30^{\text {th }}$ and pandemic on March 11th by the World Health Organization.

The complete clinical picture of COVID-19 is not fully known. Reported cases have ranged from the asymptomatic cases to the very severe illness (ARDS, heart failure, renal failure, liver damage, shock and multiorgan failure) resulting in death. Common signs of COVID-19 infection are like common cold that includes respiratory symptoms such as dry cough, sore throat, fever, breathing difficulties. In addition to respiratory failure, patients with moderate to severe illness are also likely to have coagulopathy and may predict mortality. As it is associated with high thrombotic complications which can lead to MI,stroke and microangiopathy, it is advisable and most protocols use prophylactic anticoagulation for all COVID-19 patients and full anticoagulation for certain critical ICU patients with high inflammatory markers.(LDH, D-dimer, ferritin, CRP etc). In a study by Tang et al from Wuhan, $71 \%$ of nonsurvivors from COVID-19 infection met the ISTH criteria for DIC compared to $0.4 \%$ of survivors (1). Autopsy reports showed elements of SARS COV-2, the virus causing COVID- 19, in cells lining these small blood vessels in the lungs, kidney and gut along with macro and micro thrombi ${ }^{2,5}$. It is still unclear about the mechanism involved in thrombosis but excessive inflammation, endothelial dysfunction with high levels of VWF, and coagulation Factor Viii, platelet activation, stasis and presence of lupus anticoagulant could play a role in developing hypercoagulable state in covid-19 patients. SARS-COV infection overwhelms the normally protective profibrinolytic pathway, including plasminogen activator $1^{3}$. It was found that upregulation of fibrinogen synthesis by the liver is stimulated by IL6 (4) and was present in almost all patients responsible for activation of blood coagulation with progressive D- 
dimer elevation. It is assuming that there is bi-directional synergistic relationship between DIC and cytokine storm. Studies have found that patients with D-dimer $>1000$ during admission are 20 times more likely to die than patients with lower D-dimer values ${ }^{6}$. The involvement of consumption of coagulation factors and platelets resulting in thrombocytopenia and hypo coagulant state. Though the bleeding risk is very little. According to the MOUNT SINAI, NY hospital records bleeding rate is around $3 \%$ in patient on anticoagulation compare with $2 \%$ in patient without anticoagulation but slightly higher $(7.5 \%)$ who were intubated ${ }^{7}$. Our patient who was intubated 10 days before and extubated 2 days before developing retroperitoneal bleed leading to shock and reintubation. He was initially on DVT prophylaxis and later on full anticoagulation.

Management can be challenging. In a retrospective series of 2773 individuals hospitalized with COVID-19, in whom 786 (28 percent) received systemic anticoagulation, anticoagulation was associated with improved in-hospital survival in intubated patients (71 percent, versus 37 percent for those who were not anticoagulated $^{8}$. DVT prophylaxis should be initiated for all hospitalized patients with LMWH unless the risk of bleeding is judged to exceed the risk of thrombosis along with mechanical thromboprophylaxis. Choice of anticoagulant and dose adjustment should be used per institutional guidance and renal status. Unless contraindicated full anticoagulant therapy is recommended in suspected (sudden deterioration in respiratory status or otherwise unexplained respiratory failure or physical findings consistent with thrombosis), confirmed VTE (venous thromboembolism), recurrent clotting of intravascular access devices (arterial lines, central venous catheters) despite prophylactic anticoagulation and high risk patient (critically ill patient, markedly elevated inflammatory markers, multi organ failure, D-dimer $>3000 \mathrm{ng} / \mathrm{ml}$ or Fibrinogen $>8 \mathrm{~g} / \mathrm{L}$ ). A

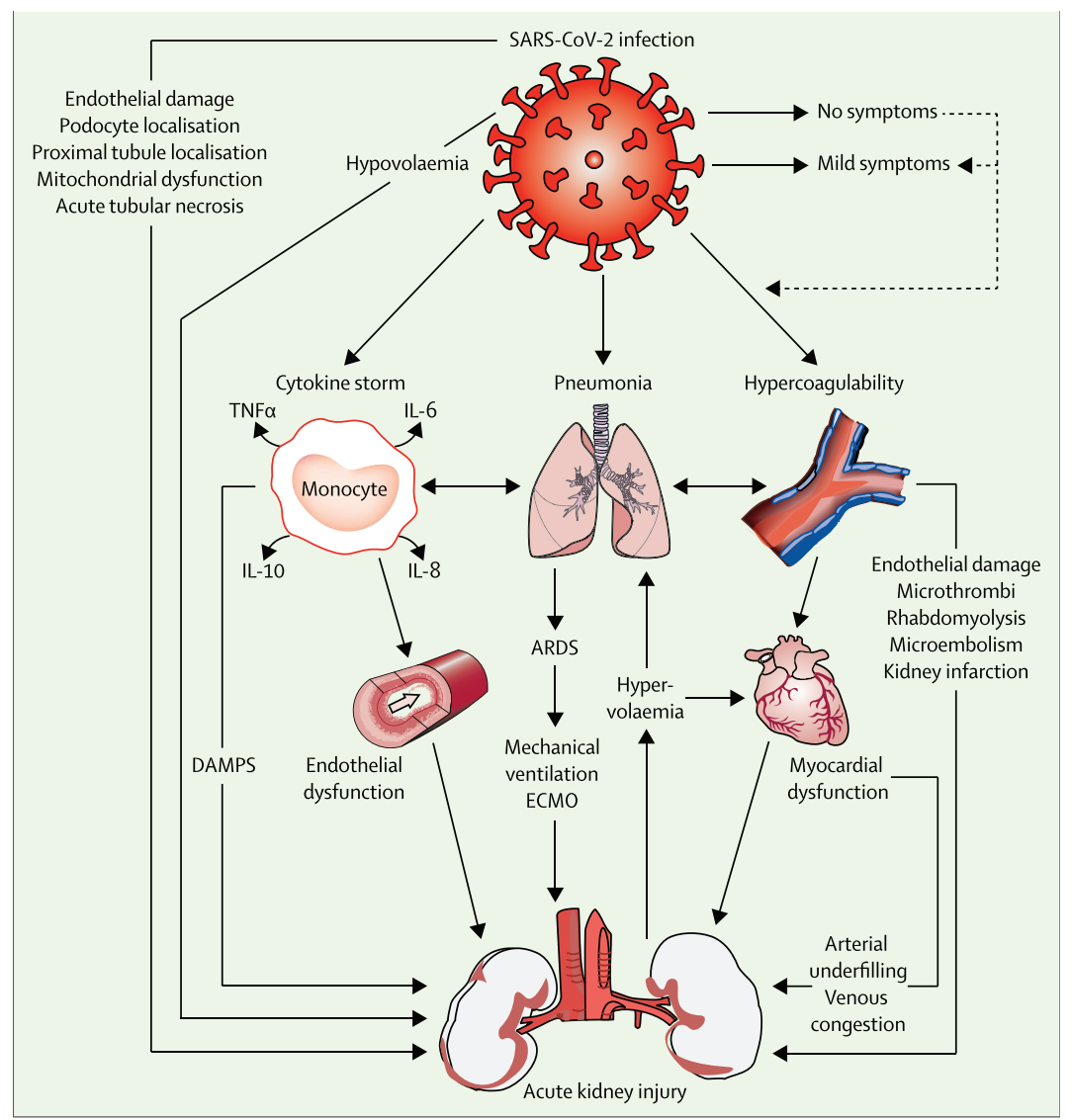

https://marlin-prod.literatumonline.com/cms/attachment/5ddb9506-2f35-474c-8b09-a62807e9621d/gr1.jpg 
prospective cohort study showed about $96 \%$ receiving CRRT ( Continuous Renal Replacement Therapy) experienced circuit clotting ${ }^{9}$. Parenteral anticoagulant especially SC enoxaparin is preferred agent. But for a patient with a history of heparin induced thrombocytopenia it may be reasonable to use fondaparinux. It is also reasonable to consider post discharge extended thromboprophylaxis (30-90 days) with DOAC (Direct-Acting Oral anticoagulant). ${ }^{10,11}$. The existing evidence on thrombotic complications and management has been based on observational, non RCT, small, retrospective analyses. Currently few randomized, open labelled, multicenter clinical trials are underway to find the detail associations and management.

Our patient also developed AKI (Cr.4.85) 4 days after intubation needed CRRT (Continuous Renal Replacement Therapy). AKI is increasingly recognized in hospitalized COVID 19 patient. Recent studies have found that in a large cohort of hospitalized patients at both tertiary care \& community hospitals, that the rates of AKI were much higher than previously reported in literatures. Studies have found that 36.6\% COVID-19 patients developed AKI during hospitalization ${ }^{12}$. They have found that AKI occurred in close temporal proximity to the time of intubation and mechanical ventilation. Among ventilated patients the rate of AKI was $89.7 \%$, compared to $21.7 \%$ among other patients, $65.5 \%$ of patients on ventilators developed stage II and stage III AKI compared to $6.7 \%$ of non-ventilated patients, and almost all of the patients requiring RRT were on ventilator support $(276 / 285)$ that is $96.8 \%{ }^{12}$. Another study showed around $20 \%$ of patients admitted to an intensive care unit (ICU) with COVID-19 require renal replacement therapy (RRT) at a median of 15 days from illness onset $^{13}$. The cause of kidney involvement in COVID-19 is likely to be multifactorial, and predisposing factors (eg, sepsis, hypovolemia, rhabdomyolysis, macrophage activation syndrome, micro thrombi and nephrotoxins) as important contributors. Another potential mechanism of AKI involves SARS-CoV-2-related immune response dysregulation.

https://marlin-prod.literatumonline.com/cms/ attachment/5ddb9506-2f35-474c-8b09-a62807e9621d/ gr1.jpg

Management of AKI is mainly supportive. COVID-19 patients admitted in ICU with AKI have a very high tendency of having respiratory failure \& sometimes it's difficult to differentiate whether it is due to volume overload or from pneumonia. Respiratory failure may be the main cause for initiation of RRT (Renal Replacement therapy) in these patients. Other indications for RRT are uremic menifestations or severe hyperkalemia ${ }^{14}$. The continous Renal Replacement Therapy (CRRT) or Intermittent Hemodialysis (IHD) are the usual methods for RRT. But convection based dialysis (CVVH,CVVHDF) which has the theoretical benefit of cytokines removal is the better choice for renal replacement therapy than conventional hemodialysis which is diffusion based therapy.Sustained Low Efficacy Dialysis (SLED) as a renal replacement modality has been increasingly used in crticially ill patients in developing country ${ }^{15}$. SLED uses standard machines, and lower dialysate and blood-flow rates over a longer time period. When any component of inpatient dialysis resources is low, urgent start peritoneal dialysis (PD) for AKI can be considered ${ }^{16}$ as an exception.It has been used in few hospitals in new york when they ran out of CRRT( Continuous Renal Replacement therapy) machines, due to high volume of critically ill patients on Renal Replacement Therapy. Outcome data are limited, but acute PD appears to be as effective as other forms of RRT in the setting of AKI when CRRT is not readily available $^{17}$.

\section{References:}

1. Tang N, Li D, Wang X, Sun Z. Abnormal Coagulation parameters are associated with poor prognosis in patients with novel coronavirus pneumonia. J Thromb Haemost. 2020. https://doi.org/10.1111/jth.14768

2. Zsuzsanna Varga et al.. Endothelial cell infection and endotheliitis in COVID-19. https://www.thelancet.com/ journals/lancet/article/PIIS0140-6736(20)30937-5/

3. High risk of thrombosis in patients with severe SARS-CoV2 infection: a multicenter prospective cohort study. https:/ /www.ncbi.nlm.nih.gov/pmc/articles/PMC7197634/

4. CL Carty,et al.Fibrinogen and IL6 Gene Variants and IL-6 Levels in Relation to Plasma Fibrinogen Concentration and Cardiovascular Disease Risk in the Cardiovascular Health Study. https://www.ncbi.nlm.nih.gov/pmc/articles/ PMC2946374/

5. leu et al. https://www.preprints.org/manuscript/ 202002.0407/v2

6. Fei Zhou MD et al. clinical course and risk factors for mortality of adult inpatients with COVID-19 in Wuhan, China: a retrospective cohort study. https:// 
www.thelancet.com/journals/lancet/article/PIIS01406736(20)30566-3/

7. pulmCrit - Thrombosis update in COVID-19: Data from the Mount Sinai system in NYC. https://emcrit.org/ pulmcrit/sinai/

8. http://www.onlinejacc.org/content/early/2020/05/05/ j.jacc.2020.05.001 (Accessed on May 07, 2020).

9. Helms $J$ et all. High risk of thrombosis in patient (with) severe SARS0CoV-2 infection.https://www.esicm.org/wpcontent/uploads/2020/04/863_author_proog.pdf.

10. COVID-19 and VTE/Anticoagulation. https://www. hematology.org/covid-19/covid-19-and-vteanticoagulation.

11. Crystal Phend. COVID-19 :Anticoagulation Recommended Even After Discharge. medpagetoday.com

12. Jamie S. Hirsch $1,2,3$ et al. ACUTE KIDNEY INJURY IN PATIENTS HOSPITALIZED WITH COVID-19 https:// www. kidney-international.org/ article/ S0085-2538 (20) 30532-9/fulltext? mobileUi $=0 \&$ fbclid $=$ IwAR2yVhqvB6v MPBrjxKFAo116 Llk11AKJ071t_rbJZb 3DD1KyCw 1 WebK137k.

13. Clinical course and risk factors for mortality of adult inpatients with COVID-19 in Wuhan, China: a retrospective cohort study. Lancet. 2020; 395: 1054-1062.

14. Advice for Managing Acute Kidney Injury in COVID-19 Patients. https://www.medscape.com/viewarticle/929275.

15. Renato A. Caires, et al. Sustained low-efficiency extended dialysis (SLED) with single-pass batch system in criticallyill patients with acute kidney injury (AKI). https:// link.springer.com/article/10.1007/s40620-015-0224-y

16. Cullis B, : Peritoneal dialysis for acute kidney injury. Perit Dial Int 34: 494-517, 2014pmid:25074995.

17. Chionh CY: Use of peritoneal dialysis in AKI: A systematic review. Clin J Am Soc Nephrol 8: 1649-1660, 2013pmid:23833316. 